\title{
A COUNTEREXAMPLE TO THE BRECKENRIDGE- MAGIDOR ACCOUNT OF INSTANTIAL REASONING
}

\author{
TRISTAN HAZE
}

THE UNIVERSITY OF SYDNEY

\begin{abstract}
A вstract: In a recent paper, Breckenridge and Magidor argue for an interesting and counterintuitive account of instantial reasoning. According to this account, in arguments such as one beginning with 'There is some $x$ such that $x$ is mortal. Let $\mathrm{O}$ be such an $x \ldots$...' the 'O' refers to a particular object, although we cannot know which. I give and defend a simple counterexample involving the notion of an unreferred-to object.
\end{abstract}

$\mathrm{I}_{\mathrm{N}}$ their (2012), Breckenridge and Magidor argue for the following thesis:

Arbitrary Reference (AR): It is possible to fix the reference of an expression arbitrarily. When we do so, the expression receives its ordinary kind of semantic-value, though we do not and cannot know which value in particular it receives.

Their primary argument in favour of AR is that it can be used to give an attractive account of "instantial reasoning" such as this (their "Argument 1"):

(1) There is someone $x$ such that for every person $y, x$ loves $y$ [Premise]

(2) Let John be such a person

(3) For every person $y$, John loves $y$ [Existential Instantiation on 1]

(4) Let Jane be an arbitrary person

(5) John loves Jane [Universal Instantiation on 3]

(6) There is some person $x$ such that $x$ loves Jane [Existential Generalisation on 5]

(7) But since Jane was an arbitrary person, for every person $y$ there is some person $x$ such that $x$ loves $y$ [Universal Generalisation on 6]

According to Breckenridge and Magidor, in this argument, the term 'John' refers to a particular person in the world, but we cannot know whom. Likewise for 'Jane.'

I think it is safe to say that this is a counterintuitive account of instantial reasoning. My purpose here is to give and defend a counterexample. It takes the form of a piece of instantial reasoning that is intuitively in order, but that leads to an absurd consequence when the Breckenridge-Magidor account of instantial reasoning is applied. 
The following is a logical truth:

(Unref) If all unreferred-to objects are white and there is an unreferred-to object, then there is a white object.

(By 'unreferred-to object,' I mean an object that is never referred to by anyone or anything.)

Here is an argument for (Unref):

(1) All unreferred-to objects are white and there is some unreferred-to object. [Assumption]

(2) All unreferred-to objects are white. [Conjunction Elimination on 1]

(3) There is some unreferred-to object. [Conjunction Elimination on 1]

(4) Let $\mathrm{O}$ be such an object.

(5) $\mathrm{O}$ is white. [Universal Instantiation on 2]

(6) There is some white object [Existential Generalization on 5]

(Unref) now follows from (1)-(6) by conditional proof.

This is a valid argument. But the theory of instantial reasoning advanced by Breckenridge and Magidor seems to imply that the expression ' $\mathrm{O}$ ' above refers to an unreferred-to object, which is absurd. I therefore suggest that this account of instantial reasoning is false.

Is there a way out for Breckenridge and Magidor? This passage from their paper, where they consider a quite different objection, according to which AR violates certain necessary conditions on reference, suggests a possible escape strategy:

It does not follow from our view that any stipulation of the form 'Let a be an arbitrary F' successfully results in fixing a referent for ' $a$ '. One could plausibly require, for example, that if there are no Fs the stipulation fails and it is perfectly compatible with AR that it also fails when other, more complex conditions, do not obtain. (379)

Could this point be used to escape the counterexample? On reflection, I think, clearly not. Firstly, the example of a stipulation failing due to there being no Fs clearly doesn't apply: there surely are unreferred-to objects (or, supposing I'm wrong about that and there are none: the argument in question would still have been valid if there had been unreferred-to objects). ${ }^{1}$ What about the possibility that the stipulation (4) fails due to the violation of some more complex condition? I think the proper response to this is simple: the argument is clearly valid, and the stipulation (4) clearly has not failed. There is no reason to think otherwise independent of a highly theoretical view about instantial reasoning such as Breckenridge and Magidor's, and our conviction that the argument is in order should trump any credence we might give to such a view.

Finally, note that while the argument I used as a counterexample involved an assumption and used conditional proof, this was not essential. I chose to frame it initially as a proof of a not-completely-trivial logical truth for rhetorical purposes 
only. (1) could be regarded as a premise, and (6) as a conclusion. Also, it is not essential that the counterexample argument begins with what is presumably a false assumption (or premise). Witness:

(1) There is some object $x$ such that $x$ is unreferred-to and $x$ is smaller than London [Premise]

(2) Let $\mathrm{O}$ be such an object.

(3) $\mathrm{O}$ is unreferred-to and $\mathrm{O}$ is smaller than London [Existential Instantiation on 1]

(4) $\mathrm{O}$ is smaller than London [Conjunction elimination on 3]

(5) There is some object $x$ such that $x$ is smaller than London.

Here, the premise and the conclusion are presumably both true.

\section{ENDNOTE}

1. It is worth noting that here we may have the seeds of another objection to the AR-based theory of instantial reasoning, since it appears that we can perform instantial reasoning with uninstantiated predicates, namely when reasoning under an assumption. I will not press this point here.

\section{BIBLIOGRAPHY}

Breckenridge, Wylie, and Ofra Magidor. 2012. “Arbitrary Reference.” Philosophical Studies 158(3): 377-400. doi: http://dx.doi.org/10.1007/s11098-010-9676-z 